\title{
Multiscale Modeling of Elastic Properties of Sustainable Concretes by Microstructural-Based Micromechanics
}

\author{
V. Zanjani Zadeh and C. P. Bobko \\ Department of Civil, Construction and Environmental Engineering, North Carolina State University, \\ Campus Box 7908, Raleigh, NC 27695-7908, USA \\ Correspondence should be addressed to C. P. Bobko; chris_bobko@ncsu.edu
}

Received 20 May 2014; Revised 29 September 2014; Accepted 30 September 2014; Published 2 November 2014

Academic Editor: Baozhong Sun

Copyright ( 2014 V. Zanjani Zadeh and C. P. Bobko. This is an open access article distributed under the Creative Commons Attribution License, which permits unrestricted use, distribution, and reproduction in any medium, provided the original work is properly cited.

\begin{abstract}
This paper addresses multiscale stiffness homogenization methodology to extract macroscale elastic mechanical properties of four types of sustainable concretes from their nanoscale mechanical properties. Nine different sustainable concrete mixtures were studied. A model based on micromechanics was used to homogenize the elastic properties. The hardened cement pastes were homogenized by three analytical methods based on Self-Consistent and Mori-Tanaka schemes. The proposed multiscale method combines advanced experimental and analytical methods in a systematic way so that the inputs are nanoscale phases properties extracted from statistical nanoindentation technique and mechanical properties of mixture ingredient. Predicted elastic properties were consistent with traditional experimental results. Linking homogenized mechanical properties of sustainable concrete to volume proportions through an analytical approach provides a critical first step towards rational optimization of these materials.
\end{abstract}

\section{Introduction}

Concrete is a complex heterogeneous material whose mechanical properties can vary substantially from point to point. Predicting the mechanical behavior of such materials always has been a challenge confronting scientists. However, progress in both experimentation and continuum micromechanics has provided the required foundations for the development of multiscale models for complex heterogeneous materials such as concrete. These upscaling schemes work as ways to exchange information about mechanical properties between different scales of a model. Furthermore, performance-oriented optimization of the homogenized properties becomes possible by considering modifications to the material chemistry, composition, processes, and their related impacts on microstructure.

\section{Background}

2.1. Sustainable Concrete. Concrete containing fly ash and blast furnace slag, kenaf fiber reinforced concrete (KFRC), and lightweight aggregate concrete (LWAC) can be categorized as sustainable concrete, by achieving concrete with high strength while reducing cement consumption. Plant-based natural fibers, such as kenaf bast fibers, can be used in concrete to obtain a new lighter weight and yet tougher concrete while maintaining desirable material properties will directly enhance the merits of precast concrete [1]. $\mathrm{CO}_{2}$ sequestration is another positive impact of kenaf. One acre of kenaf captures as much $\mathrm{CO}_{2}$ as three acres of rain forest, and much of the captured $\mathrm{CO}_{2}$ can be permanently trapped inside structures used in construction [2]. Additionally, the lighter weight and better performance will reduce the energy requirements and greenhouse gas emissions associated with concrete manufacturing and delivery.

Using fly ash (FA) and ground granular blast furnace slag (GGBS) reduces greenhouse gas emissions, since unlike Portland cement their reaction with water does not produce $\mathrm{CO}_{2}$. In addition to reducing $\mathrm{CO}_{2}$ emissions, other potential benefits of using FA and GGBS include their disposal costs, significant opportunity to reduce landfill volumes, manufacturing energy savings, lower-cost concrete, and reduction of water needed to produce the concrete $[3,4]$. 
Being much lighter than conventional aggregates, due to cellular or highly porous microstructure, lightweight aggregate (LWA) contributes to improved sustainability of concrete by reducing transportation cost [5]. Also, LWAs have better fire resistance properties compared to conventional aggregates [6]. Furthermore, LWAs often are made of waste materials during a high temperature baking, which lessen the need of using naturally occurring mineral aggregates, although tradeoffs in energy consumption during the manufacturing process must be weighed against the benefits. Besides direct contribution of LWA to sustainability, it may have a positive effect on mechanical properties of concrete. The use of internal curing generated by LWA can substantially mitigate self-desiccation and resulting autogenous deformation and cracking thus reducing transport properties such as diffusion and sorptivity [5]. This may increase the service life of concrete structures through more resistance against water permeability and freeze-thaw damage. Also, the enhanced hydration and increased compressive strengths provided by internal curing specifically in interfacial transition zone (ITZ), the volume surrounding aggregates, may allow for small but significant reductions in cement content in many concrete mixtures $[7,8]$. All these benefits of LWA can compensate the energy use during LWA production and can contribute to significant improvements in sustainability.

2.2. Concrete Constituents and Microstructure. Inspired by the elastic properties model proposed by Constantinides and Ulm [9], we introduce a four-level multiscale model for the sustainable concretes (KFRC and concrete containing lightweight aggregate, fly ash, and slag) considered in this research. The bottom-up models for the mixtures are displayed in Figure 1 in detail. The 2D sketches refer to $3 \mathrm{D}$ volumetric elements, and shapes in the illustrations serve to identify phases, not to indicate specific morphologies. $L$ represents the REV size. Each level is separated from the next one by one order of magnitude in size of the elementary heterogeneity. In this multiscale model, each scale verifies the scale separability condition which is expressed in theory of homogenization as

$$
d_{0} \ll d \ll D \ll L, \quad l \ll \lambda,
$$

where $d_{0}$ is the smallest length scale that continuum mechanics is not valid, $d$ denotes the characteristic size of the inhomogeneities or deformation mechanism, $D$ is the REV size, $L$ is the dimension of the whole body of material, and $\lambda$ is the fluctuation length of the material properties.

Hydrated cement paste at submicron scale level is the foundation for the multiscale approach in this study. Approximately $70 \%$ of fully hydrated cement paste consists of calcium-silicate-hydrate (C-S-H) gel, $20 \%$ calcium hydroxide $(\mathrm{CH})$, and the rest is ettringite, calcium monosulfate aluminate, unhydrated clinker residue, and other minor constituents [10]. Recent studies by Ulm and coworkers $[11,12]$ have led to a useful model suggesting that there are three types of C-S-H (LD C-S-H, HD C-S-H, CH/C$\mathrm{S}-\mathrm{H}$ nanocomposite) in hardened cement paste. Together with another research by Jennings [13] the model proposes

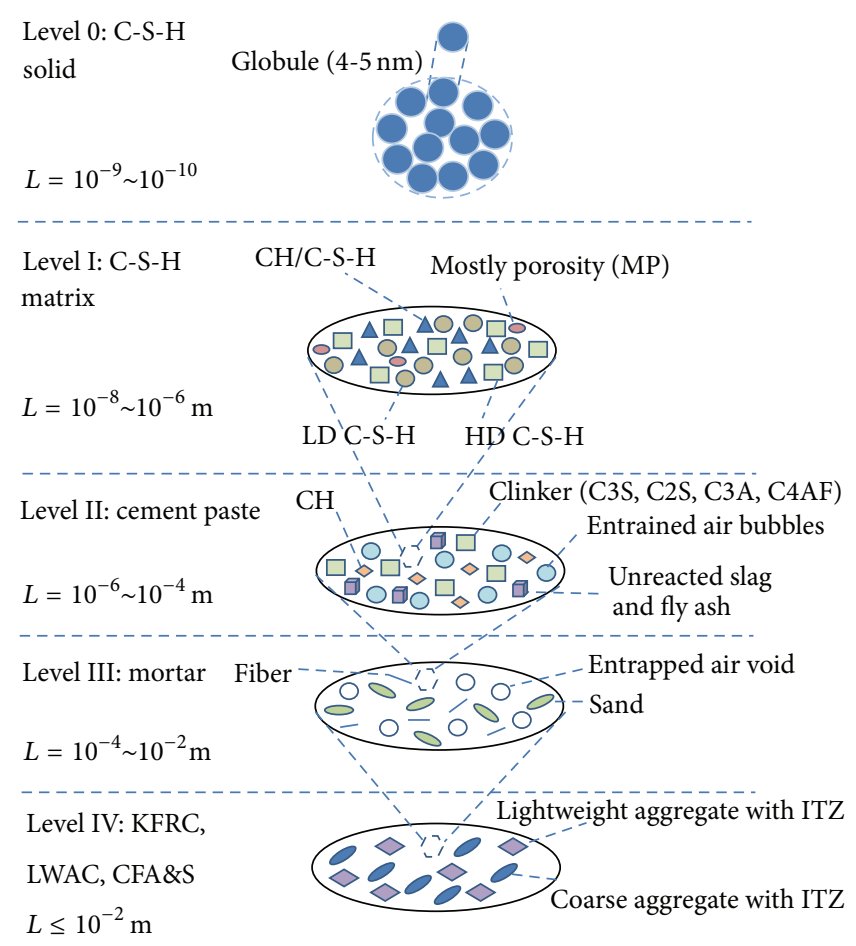

FIGURE 1: Bottom-up four-scale level model for the samples.

that LD C-S-H and HD C-S-H are composed of similar elementary particles with different nanoscale porosity, while $\mathrm{CH} / \mathrm{C}-\mathrm{S}-\mathrm{H}$ phase is comprised of a nanocomposite of C-S$\mathrm{H}$ and $\mathrm{CH}$ [12]. The average nanoscale material responses (e.g., indentation modulus and indentation hardness) for each of these three C-S-H phases are virtually the same for all Portland cement pastes tested to date [11, 14].

In the four-scale level model established in this study, level I spans the characteristic length between $10 \mathrm{~nm}-1 \mu \mathrm{m}$, which is comprised of medium and large capillary porosity and most of the hydration products $(70-80 \%$ of hydration products) which are the C-S-H morphologies. This scale level explicitly differentiates LD C-S-H, HD C-S-H, and CH/C-S$\mathrm{H}$ phases.

In the scale level II which is considered at length scale between $1 \mu \mathrm{m}-100 \mu \mathrm{m}$, homogenized C-S-H matrix, clinker, rejected fly ash and slag, gypsum, $\mathrm{CH}$, and large capillary porosity are found.

Scale level III refers to mortar and is assumed on the characteristic length scale of $1 \mathrm{~mm}-1 \mathrm{~cm}$. It contains homogenized cement paste, sand or fine aggregates, fiber, associated ITZs, and air voids.

The largest scale level IV is considered on the scale level of $1 \mathrm{~cm}-10 \mathrm{~cm}$. This macroscopic scale includes all the ingredients of concrete and is the classical scale of material testing of concrete. Mortar, lightweight aggregate, coarse aggregate, and associated ITZ are typically found at this scale.

2.3. Multiscale Modeling. Traditionally, multiscale modeling of heterogeneous materials where the phases are clearly separated is executed through homogenization methods. Micromechanics based on a simple definition of eigenstrain 
in an inclusion came with Eshelby's equivalent homogenization theory [15-17] and Hashin's variational principle [18, 19]. In 1960s, the early Voigt [20], Reuss [21], and Taylor [22] estimates were reassessed as bounds which became the basis for so-called continuum micromechanics [23]. Effective field theories based on the Eshelby elasticity solution with different assumptions were developed for inhomogeneities embedded in infinite media [24-26]. New developments have been made continuously, with special mention of refined boundaries associated with an improved morphological description and by means of special cases of media with periodic microstructures [27-29].

Concrete is a highly heterogeneous composite construction material whose microstructure contains randomly dispersed features. The heterogeneity of concrete exists in a variety of length scales from nano to macro. It is widely recognized that many macroscopic phenomena of the concrete originate from the mechanics of the underlying nano- and microscale structure. All the mechanical, physical, and chemical properties of the ingredients including stiffness, strength, size, shape, volume fraction, and spatial distribution can have impact on the macroscale properties. The homogenization focuses on multiscale analysis due to the fact that typical material phases may be found at separable length scales as shown in Figure 1. Several multiscale micromechanical modeling techniques have been suggested for obtaining macroscopic properties (elasticity, strength, etc.) of concrete from knowledge of its nanoscale constituent properties, including both numerical (e.g., [30]) and analytical [31-34] approaches.

In this paper, multiscale modeling of mechanical properties of four types of sustainable concretes is presented. A multistep, multiscale analytical micromechanics model is defined based on morphology and length scale of the structural elements in each material. Unlike the previous analytical models that were proposed and used only in theoretical problems or for samples that were built in the laboratory, in this paper the multiscale model was used for sustainable concretes including fly ash and blast furnace slag that were poured and built in real condition. This is a leap forward in applying multiscale modeling techniques to the sustainable concrete industry instead of being used only in laboratory. The upscaled outcome of the modeling is compared with traditional macroscopic results and they are found to be consistent. Comparing the model prediction results with macroscopic experimental data permits the validation of the applied homogenization models. The validation itself can be employed to validate the measured mechanical properties and morphology of the building blocks at the smallest length scales. Results from this research may help development of guidelines that aim at optimizing mix designs of cementitious materials with kenaf, LWA, and fly ash/slag for the desired mechanical properties.

\section{Effective Elastic Modulus of Heterogeneous Materials}

A three-step homogenization scheme is developed to bridge the four length scale levels in order to obtain elasticity constants of the samples in macroscopic scale from knowledge of the volume fractions. Continuum micromechanics offers a framework to address this challenge.

Continuum micromechanics relies on the concept of a concentration tensor which is able to bridge the gap between local stress and strain fields $(\sigma, \varepsilon)$ from macroscopic ones $(\Sigma$, E) as follows:

$$
\Sigma=\mathbf{A}: \sigma, \quad E=\mathbf{B}: \varepsilon,
$$

in which $\mathbf{A}$ and $\mathbf{B}$ are fourth-order stain and stress localization (concentration) tensors.

Consider an heterogeneous material $(L)$ is made of a matrix phase $\varphi_{0}$ and a set of $N$ inhomogeneities that can be described as inclusions of similar ellipsoidal shape, $\varphi_{1}, \varphi_{2}, \ldots, \varphi_{N}$ which is displayed in Figure 2. Also consider elasticity and compliance tensors of the solid composite which are represented as $\mathbf{C}$ and $\mathbf{S}$, respectively.

Clearly enough, the volume fraction of $j$ th inhomogeneity $\left(\varphi_{j}\right)$ is defined as $f_{j}=\varphi_{j} / \varphi$, and volume fraction of different phases sums to one:

$$
\sum_{j=0}^{N} f_{j}=1 .
$$

Also, assume the stiffness tensor of $\varphi_{j}$ is $\mathbf{c}_{j}(j=0,1, \ldots$, $N)$. The goal of elastic homogenization is finding stiffness tensor of the composite in terms of the stiffness tensors of the matrix and inhomogeneities [35]. The concentration tensor is critical, because once such concentration tensors are found, the composite effective mechanical properties can be attained. Generally, this problem cannot be solved without additional assumptions, because boundary condition of inclusions is unknown. Therefore, in order to solve the problem, it has to be transformed to a homogenous boundary condition problem. It is supposed that the inhomogeneous solid is subjected to either displacement or traction boundary condition. In the case of traction boundary condition, the boundary condition will be in the form of

$$
p(x)=\Sigma \cdot n, \quad \forall n \in V,
$$

where $n$ is the unit vector outward at the boundary and $p$ is surface traction. Then for any equilibrated stress field, the average stress of the REV equals the homogenous stress or macroscopic stress in domain $V$ :

$$
\langle\sigma\rangle=\frac{1}{V} \int_{V} \sigma(x) d V=\Sigma .
$$

Similarly, macroscopic strain can be imposed through following so-called displacement boundary condition of the Hashin type:

$$
\varepsilon(x)=E \cdot x, \quad \forall x \in V,
$$

where $E$ denotes the macroscopic strain which maps position $x$ on microscopic strain $\varepsilon(x)$. For any strain field derived from any compatible strain field, the macroscopic strain is expressed as

$$
\langle\varepsilon\rangle=\frac{1}{V} \int_{V} \varepsilon(x) d V=E .
$$




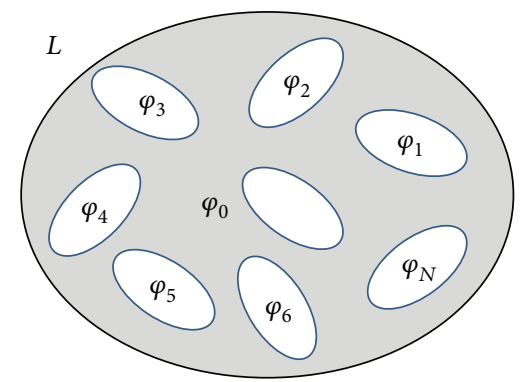

Figure 2: Composite material with $N$ inhomogeneities.

The strain average rule, $E=\bar{\varepsilon}$, implies that $\overline{\mathbf{A}}=\mathbf{I}$. Figure 3 shows a composite material with $N$ randomly oriented inhomogeneities. Considering $j$ th inhomogeneity and its interaction with surrounding matrix and other inhomogeneities, the stiffness of the matrix around the inhomogeneity is not same as the matrix stiffness $\left(\mathbf{c}_{0}\right)$. For the same reasons, it is plausible to attribute different matrix strain in inhomogeneity neighborhood. Let this unknown matrix stiffness and strain be called $\widehat{\mathbf{c}}_{0}$ and $\widehat{\varepsilon}_{0}$. Now an inclusion problem is generated to simulate the inhomogeneity problem. By introducing an eigenstrain $\varepsilon^{*}$ and adjusting it, using Hooke's law we can equate stress fields on the inhomogeneity with equivalent inclusion equation (total stress in $L$ and $L-\varphi$ ).

Total strain in inhomogeneity can be written as

$$
\begin{gathered}
\varepsilon_{j}=\left(\widehat{\varepsilon}_{0}+\varepsilon_{j}^{p}\right), \\
\mathbf{c}_{j}:\left(\widehat{\varepsilon}_{0}+\varepsilon_{j}^{p}\right)=\widehat{\mathbf{c}}_{0}:\left(\widehat{\varepsilon}_{0}+\varepsilon_{j}^{p}-\varepsilon_{j}^{*}\right),
\end{gathered}
$$

where $\varepsilon_{j}^{p}$ is the perturbed strain field due to the presence of other inhomogeneities and $\mathbf{c}_{j}$ is the stiffness of a single phase. We can express the induced strain field in terms of the prescribed eigenstrain through the following relation:

$$
\varepsilon_{j}^{p}=\widehat{\mathbf{S}}_{j}^{\mathrm{Esh}}: \varepsilon_{j}^{*},
$$

where $\widehat{\mathbf{S}}_{j}^{\text {Esh }}$ is the Eshelby tensor computed using the elastic constants of $\widehat{\mathbf{c}}_{0}$ and geometry of the $j$ th inhomogeneity $\left(\varphi_{j}\right)$. Closed-form solutions for $\widehat{\mathbf{S}}_{j}^{\text {Esh }}$ are available for ellipsoidal inclusions. The spherical inclusion assumption will eliminate introducing anisotropy to the problem. The equivalent inclusion equation should be solved for eigenstrain $\varepsilon_{j}^{*}$ :

$$
\begin{gathered}
\mathbf{c}_{j}:\left(\widehat{\varepsilon}_{0}+\widehat{\boldsymbol{S}}_{j}^{\text {Esh }}: \varepsilon_{j}^{*}\right)=\widehat{\mathbf{c}}_{0}:\left(\widehat{\varepsilon}_{0}+\widehat{\mathbf{S}}_{j}^{\text {Esh }}: \varepsilon_{j}^{*}-\varepsilon_{j}^{*}\right), \\
\mathbf{c}_{j}: \widehat{\varepsilon}_{0}+\mathbf{c}_{j}: \widehat{\boldsymbol{S}}_{j}^{\text {Esh }}: \varepsilon_{j}^{*}=\widehat{\mathbf{c}}_{0}: \widehat{\varepsilon}_{0}+\widehat{\mathbf{c}}_{0}: \widehat{\mathbf{S}}_{j}^{\mathrm{Esh}}: \varepsilon_{j}^{*}-\widehat{\mathbf{c}}_{0}: \varepsilon_{j}^{*}, \\
\varepsilon_{j}^{*}=-\left[\left(\mathbf{c}_{j}-\widehat{\mathbf{c}}_{0}\right): \widehat{\boldsymbol{S}}_{j}^{\mathrm{Esh}}+\widehat{\mathbf{c}}_{0}\right]^{-1}\left(\mathbf{c}_{j}-\widehat{\mathbf{c}}_{0}\right): \widehat{\varepsilon}_{0} .
\end{gathered}
$$

Therefore by substituting (10) in (8) total strain in the $j$ th inhomogeneity will be found:

$$
\begin{aligned}
\varepsilon_{j} & =\widehat{\varepsilon}_{0}+\varepsilon_{0}^{p}=\widehat{\varepsilon}_{0}+\widehat{\mathbf{S}}_{j}^{\text {Esh }}: \varepsilon_{j}^{*} \\
& =\left[\mathbf{I}-\left[\left(\mathbf{c}_{j}-\widehat{\mathbf{c}}_{0}\right): \widehat{\mathbf{S}}_{j}^{\text {Esh }}+\widehat{\mathbf{c}}_{0}\right]^{-1}:\left(\mathbf{c}_{j}-\widehat{\mathbf{c}}_{0}\right)\right]: \widehat{\varepsilon}_{0} .
\end{aligned}
$$

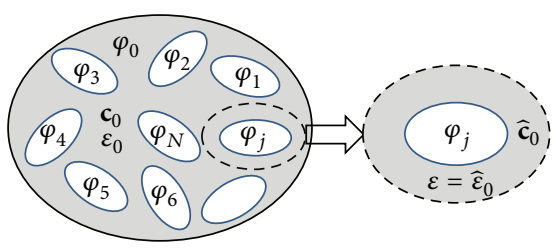

FIgURE 3: The $j$ th inhomogeneity in the composite.

And after some transformation,

$$
\begin{aligned}
= & {\left[\widehat{\mathbf{s}}_{j}^{\text {Esh }}+\left(\mathbf{c}_{j}-\widehat{\mathbf{c}}_{0}\right)^{-1}: \widehat{\mathbf{c}}_{0}-\widehat{\mathbf{s}}_{j}^{\text {Esh }}\right] } \\
& \times\left[\widehat{\mathbf{S}}_{j}^{\text {Esh }}+\left(\mathbf{c}_{j}-\widehat{\mathbf{c}}_{0}\right)^{-1}: \widehat{\mathbf{c}}_{0}\right]^{-1}: \widehat{\varepsilon}_{0} \\
= & \left(\mathbf{c}_{j}-\widehat{\mathbf{c}}_{0}\right)^{-1}: \widehat{\mathbf{c}}_{0}:\left[\widehat{\mathbf{S}}_{j}^{\text {Esh }}+\left(\mathbf{c}_{j}-\widehat{\mathbf{c}}_{0}\right)^{-1}: \widehat{\mathbf{c}}_{0}\right]^{-1}: \widehat{\varepsilon}_{0} \\
= & \left(\left[\widehat{\mathbf{S}}_{j}^{\text {Esh }}+\left(\mathbf{c}_{j}-\widehat{\mathbf{c}}_{0}\right)^{-1}: \widehat{\mathbf{c}}_{0}\right]^{-1}: \widehat{\mathbf{c}}_{0}^{-1}:\left(\mathbf{c}_{j}-\widehat{\mathbf{c}}_{0}\right)^{-1}\right)^{-1}: \widehat{\varepsilon}_{0} \\
= & {\left[\widehat{\mathbf{S}}_{j}^{\text {Esh }}: \mathbf{c}_{0}^{-1}:\left(\mathbf{c}_{j}-\widehat{\mathbf{c}}_{0}\right)+\mathbf{I}\right]^{-1}: \widehat{\varepsilon}_{0} } \\
= & {\left[I+\widehat{\boldsymbol{S}}_{j}^{\text {Esh }}:\left(\mathbf{c}_{0}^{-1}: \mathbf{c}_{j}-\mathbf{I}\right)\right]^{-1}: \widehat{\varepsilon}_{0}=\widehat{\mathbf{H}}_{j}: \widehat{\varepsilon}_{0}, }
\end{aligned}
$$

where $\widehat{\mathbf{H}}_{j}$ is the fourth-order local strain localization tensor for $j$ th inhomogeneity. This matrix relates strain in the inhomogeneity to any strain tensor. The expression of the corresponding stress similarly can be derived:

$$
\sigma_{j}=\mathbf{c}_{j}: \varepsilon_{j}=\mathbf{c}_{j}: \widehat{\mathbf{H}}_{j}: \widehat{\varepsilon}_{0} .
$$

Now stress and strain field in $j$ th inhomogeneity is related to an unknown strain field $\left(\widehat{\varepsilon}_{0}\right)$ and fabricated matrix stiffness $\left(\widehat{\mathbf{c}}_{0}\right)$. Once $\widehat{\varepsilon}_{0}$ and $\widehat{\mathbf{c}}_{0}$ are known, the local concentration tensor could be computed, so that the effective mechanical properties of composite can be found.

3.1. Mori-Tanaka Scheme. The Mori-Tanaka (MT) scheme [26] is one of the commonly applied homogenization procedures in micromechanics. Despite stress and strain fields that may be different around each inhomogeneity, one can find average stress and strain fields $\left(\bar{\sigma}_{0}, \bar{\varepsilon}_{0}\right)$ in the matrix that can suitably represent stress and strain fields in the inhomogeneities neighborhood suitably. Also, it can be assumed that by removing one inhomogeneity the average stress and strain are the same. Figure 4 shows the illustration of assumption for $j$ th inhomogeneity in MT method.

The assumptions can be written as

$$
\widehat{\mathbf{c}}_{0}=\mathbf{c}_{0} \quad \widehat{\varepsilon}_{0}=\bar{\varepsilon}_{0} \quad \widehat{\mathbf{S}}_{j}^{\text {Esh }}=\mathbf{S}_{j}^{\text {Esh }} .
$$

Using these assumptions, the global strain localization tensor can be found as it relates global strain to strain in inhomogeneities:

$$
\mathbf{A}_{j}=\mathbf{a}_{j}\left[f_{0} \mathbf{I}+\sum_{i=1}^{N} f_{i} \mathbf{a}_{i}\right]^{-1}=\mathbf{H}_{j}:\left[\sum_{i=0}^{N} f_{i} \mathbf{H}_{i}\right]^{-1} .
$$




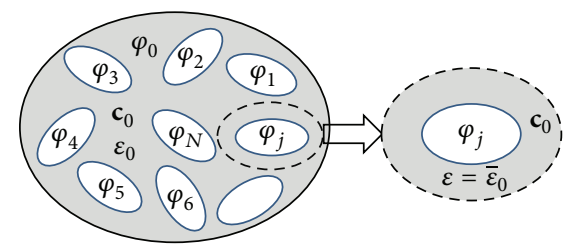

FIGURE 4: Schematic illustration of assumption for $j$ th inhomogeneity in MT method.

Because of the matrix-inclusion assumption in which matrix stiffness is a reference medium unchanged by presence of inclusions, one can have $\mathbf{H}_{0}=\mathbf{I}$. By knowing this, the effective stiffness matrix of composite will be in the form of

$$
\begin{aligned}
& \mathbf{C}_{\mathrm{MT}}^{\mathrm{est}}= \mathbf{c}_{0}+\sum_{j=1}^{N} f_{j}\left(\mathbf{c}_{j}-\mathbf{c}_{0}\right): \mathbf{A}_{j} \\
&= \mathbf{c}_{0}+\sum_{j=1}^{N} f_{j}\left(\mathbf{c}_{j}-\mathbf{c}_{0}\right): \mathbf{H}_{j}:\left[\sum_{i=0}^{N} f_{i} \mathbf{H}_{i}\right]^{-1} \\
&= \mathbf{c}_{0}+\sum_{j=1}^{N} f_{j} \mathbf{c}_{j}: \mathbf{H}_{j}:\left[\sum_{i=0}^{N} f_{i} \mathbf{H}_{i}\right]^{-1} \\
&-\mathbf{c}_{0} \sum_{i=1}^{N} f_{j}: \mathbf{H}_{j}:\left[\sum_{i=0}^{N} f_{i} \mathbf{H}_{i}\right]^{-1} \\
&= \sum_{j=1}^{N} f_{j} \mathbf{c}_{j}: \mathbf{H}_{j}:\left[\sum_{i=0}^{N} f_{i} \mathbf{H}_{i}\right]^{-1}, \\
& \mathbf{C}_{\mathrm{MT}}^{\mathrm{est}}=\sum_{j=1}^{N} f_{j} \mathbf{c}_{j}: \mathbf{H}_{j}:\left[\sum_{i=0}^{N} f_{i} \mathbf{H}_{i}\right]^{-1} .
\end{aligned}
$$

This is the MT estimate of macroscopic stiffness tensor.

Again using the MT method for a composite with isotropic matrix and randomly distributed isotropic inhomogeneity phases, the homogenized bulk and shear modulus can be further simplified [35]:

$$
\begin{gathered}
\bar{K}=K_{0}+\frac{f_{1}\left(K_{1}-K_{0}\right)\left(3 K_{0}+4 \mu_{0}\right)}{3 K_{1}+4 \mu_{0}+3\left(1-f_{1}\right)\left(K_{1}-K_{0}\right)}, \\
\bar{\mu}=\mu_{0}+\frac{5 f_{1} \mu_{0}\left(\mu_{1}-\mu_{0}\right)\left(3 K_{0}+4 \mu_{0}\right)}{5 \mu_{0}\left(3 K_{0}+4 \mu_{0}\right)+6\left(1-f_{1}\right)\left(\mu_{1}-\mu_{0}\right)\left(K_{0}+2 \mu_{0}\right)} .
\end{gathered}
$$

The 0 and 1 indices indicate the properties for matrix and inclusion, respectively. Also, $G$ and $K$ are the shear modulus and bulk modulus, respectively, and can be directly linked with elastic modulus $(E)$ and Poisson's ratio $(v)$ defined by linear isotropic elasticity:

$$
E=3 K(1-2 v), \quad v=\frac{3 K-2 G}{2(3 K-G)} .
$$

3.2. Self-Consistent Scheme. Unlike the MT scheme, in the Self-Consistent (SC) method [23] it is assumed that the $j$ th inhomogeneity is embedded in a homogenous stiffness matrix $\left(\mathbf{C}^{\text {est }}\right)$ that has been subjected to the macroscopic strain tensor $(\Sigma)$. The assumption for the $j$ th inhomogeneity in SC scheme is shown in Figure 5 schematically. This scheme is suitable for materials such as polycrystals or granular composites whose phases are dispersed in REV, and none of them plays any specific morphological role. The SC scheme implies the following assumptions:

$$
\widehat{\mathbf{c}}_{0}=\mathbf{C} \quad \widehat{\varepsilon}_{0}=E \quad \widehat{\mathbf{S}}_{j}^{E s h}=\overline{\mathbf{S}}_{j}^{\text {Esh }} .
$$

Using these assumptions, the homogenized stiffness matrix for SC scheme will be in the form of

$$
\mathbf{C}_{\mathrm{SC}}^{\mathrm{est}}=\sum_{j=1}^{N} f_{j} \mathbf{c}_{j}:\left[\mathbf{S}_{j}^{\mathrm{Esh}}: \mathbf{C}_{\mathrm{SC}}^{\mathrm{est}-1}:\left(\mathbf{c}_{j}-\mathbf{C}_{\mathrm{SC}}^{\mathrm{est}}\right)+\mathbf{I}\right]^{-1} \text {. }
$$

As demonstrated above, the SC scheme is an implicit method and typically numerical iteration is needed to solve these equations. In order to simplify the calculation of global stiffness matrix, Hashin and Shtrikman $[18,19]$ derived an elastic model for two material phases in the form of coated isotropic spheres. In case of $n$ spherical inclusions embedded in an unbounded matrix subjected to pure dilatational deformation, effective properties of the composite were derived by Herve and Zaoui [36]. Continuous strain and stress are assumed between adjacent spheres. Geometrical illustration of the Herve-Zaoui scheme is exhibited in Figure 6.

This scheme can be applied to obtain elastic properties of homogenized media. Using this scheme the SC homogenized bulk modulus will be simplified as follows [35]:

$$
\overline{K_{n}}=K_{n}+\frac{\left(f_{n-1}^{3} / f_{n}^{3}\right)\left(\bar{K}_{n-1}-K_{n}\right)\left(3 K_{n}+4 \mu_{n}\right)}{3 K_{n}+4 \mu_{n}+3\left(1-f_{n-1}^{3} / f_{n}^{3}\right)\left(\bar{K}_{n-1}-K_{n}\right)} .
$$

In which $\mu$ and $K$ are the shear modulus and bulk modulus, respectively. The indices indicate the outside layer and layered inclusions of phase, $n$ and $(n-1)$, respectively.

3.3. Implementation for the Sustainable Concretes. A threestep homogenization scheme was applied to bridge the four scale levels, as shown in Figure 1, in order to attain elasticity constants of the samples in macroscopic scale.

Scale level I explicitly differentiates the LD C-S-H, HD $\mathrm{C}-\mathrm{S}-\mathrm{H}$, and $\mathrm{CH} / \mathrm{C}-\mathrm{S}-\mathrm{H}$ phases. By considering the volume fractions of each hydration phase in the samples, as well as SEM observations, it was found out that there is not any dominant phase in this scale level. Instead, the phases could be considered granular, and the SC scheme with spherical particles was more appropriate to determine the homogenized stiffness tensor. Homogenization of these phases results in the effective mechanical properties of most of the hydrated products which is the so-called C-S-H matrix.

In the scale level II, homogenization of C-S-H matrix, clinker, rejected fly ash and slag, gypsum, $\mathrm{CH}$, and large 


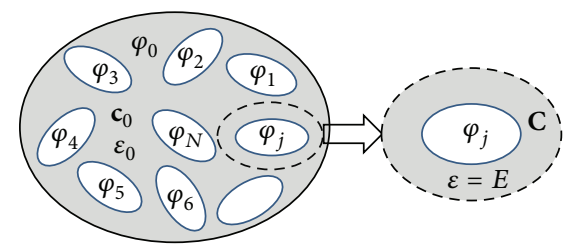

FIGURE 5: Schematic illustration of assumption for $j$ th inhomogeneity in SC method.

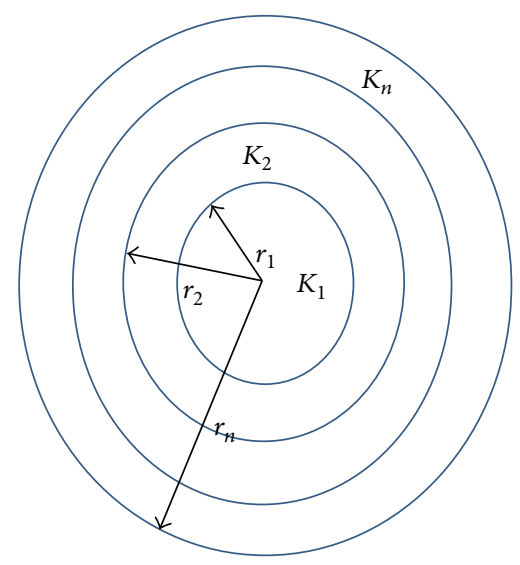

FIGURE 6: Geometrical illustration of multicoated spheres composite.

capillary porosity yielded effective mechanical properties of so-called cement paste. Since unhydrated clinker, $\mathrm{CH}$, and large capillary porosities could be assumed as inclusions that were embedded in continuous C-S-H matrix, MT scheme was appropriate to obtain the homogenized stiffness tensor. For simplicity, spherical inclusion morphologies are assumed.

In scale level III, stiffness homogenization of cement paste, sand, fiber, associated ITZs, and air void produced effective stiffness tensor of so-called mortar. For simplicity, ITZ was modeled as a randomly distributed spherical inclusion in the matrix. Again the MT scheme was the most appropriate to obtain the homogenized stiffness tensor.

Finally, in the scale level IV, stiffness of mortar, lightweight aggregate, coarse aggregate, and associated ITZ were homogenized to obtain macroscopic elastic properties. Similar to previous scale level, lightweight and coarse aggregates and their ITZs were considered as inclusions in matrix built up by mortar. MT scheme is again the appropriate method to use for properties homogenization in this scale level.

Considering the ITZ thickness around aggregates and sands is a function of aggregate size, water absorbent property of the aggregate, age, and $\mathrm{w} / \mathrm{c}$ ratio; it can be between $10 \mu \mathrm{m}$ to $80 \mu \mathrm{m}$ [37]. For the kenaf samples, the ITZ thickness was observed to be about $30 \mu \mathrm{m}$ [38]. Simple calculation shows that, by assuming $30 \mu \mathrm{m}$ and $10 \mu \mathrm{m}$ thicknesses of ITZ for coarse aggregate and sand, total volume fraction of ITZ will be less than $1 \%$. Therefore ITZ of sand and coarse aggregates did not have significant effect on predictions of macroscopic elastic properties. However, weakness of ITZ can affect fracture and strength properties of concrete which cannot be observed in this type of modeling.

The intent of the multiscale modeling procedure is to predict elastic modulus from knowledge of the mixture proportions of the concrete and elastic properties of each component of the concrete, without the need for petrographic investigation of each sample. This implies, however, that any sample defects or microcracking are not considered and some overprediction of elastic properties could be expected.

\section{Materials and Mixture Proportions}

Nine different mixtures were studied. The mixture properties and sample designations are outlined in Table 1. Large scale specimens from these mixtures were prepared and built as discussed by Elsaid et al. [2] and McCoy et al. [39] to conduct compressive strength, splitting tensile strength, and rupture experiments. KF1 and KF2 had $1.2 \%$ and $2.4 \%$ of kenaf bast fibers by volume and $\mathrm{KC}$ is made of plain concrete without fiber from the same batch. In CF2 and LF2 the total Portland cement (Type II) in the mixture was replaced by $20 \%$ fly ash (Class F), in CF6 and LF6 by $60 \%$ fly ash (Class F), and in CS6 and LS6 by $60 \%$ slag cement (grade 100) by weight. $20 \%$ fly ash replacement and $60 \%$ slag cement replacement are quantities often found in commercial construction while 60\% fly ash is quite high but could be considered for enhanced sustainability. LF2, LF6, and LS6 have the similar type of mixture properties as CF2, CF6, and CS6, respectively, except they had higher amount of sand, and conventional course aggregate (CCA) was replaced by dry expanded slate LWA (Stalite).

The volume fractions of hydration products, obtained from statistical nanoindentation technique, of the all the samples and kenaf ITZ are summarized in Figure 7 [7, 8, 38, 40].

The C-S-H hydration products, with volume fractions shown in Figure 7, form the first scale level in multiscale modeling. The rest of the scale levels include different inclusions depending on the sample's mixture (i.e., kenaf fibers, coarse or lightweight aggregates, etc.) with mix proportions listed in Table 1. Elastic properties of the concrete ingredients used in the modeling taken from open literature are listed in Table 2.

According to Mehta and Monteiro [41] total $\mathrm{CH}$ volume fraction in normal concretes is around $20 \%$ of total hydration products. For KFRC samples (normal Portland cement concrete) total $\mathrm{CH}$ volume fraction was assumed to be $20 \%$, whereas all $\mathrm{CH}$ was consumed by secondary reaction in the rest of the samples $[8,40]$. In KFRC samples, part of the $\mathrm{CH}$ was considered crystal embedded in $\mathrm{CH} / \mathrm{C}-\mathrm{S}-\mathrm{H}$ nanocomposite ( $\mathrm{CH}$ in $\mathrm{HD}$ C-S-H porosity) and the rest appeared as a separate phase in scale level II. The amount of $\mathrm{CH}$ in each scale depends on the $\mathrm{CH} / \mathrm{C}$-S-H nanocomposite volume fraction and associated gel porosity ( $24 \%$ of solid). Below equations were used to find the volume fractions of $\mathrm{CH}$ in levels I and II:

$$
\begin{gathered}
f_{\mathrm{CH}}(\text { Level I })=f_{\mathrm{HD} \mathrm{C}-\mathrm{S}-\mathrm{H}} \times \text { Gel porosity volume }(0.24), \\
f_{\mathrm{CH}}(\text { Level II })=0.20-f_{\mathrm{CH}}(\text { Level I }) .
\end{gathered}
$$


TABLE 1: Mixture proportions.

\begin{tabular}{|c|c|c|c|c|c|c|c|c|c|}
\hline Sample & $\begin{array}{l}\text { Fiber content } \\
\qquad\left(\mathrm{kg} / \mathrm{m}^{3}\right)\end{array}$ & $\begin{array}{l}\text { Portland } \\
\text { cement } \\
\left(\mathrm{kg} / \mathrm{m}^{3}\right)\end{array}$ & $\begin{array}{l}\text { Fly ash } \\
\left(\mathrm{kg} / \mathrm{m}^{3}\right)\end{array}$ & $\begin{array}{l}\text { Slag cement } \\
\quad\left(\mathrm{kg} / \mathrm{m}^{3}\right)\end{array}$ & $\begin{array}{l}\text { Coarse } \\
\text { aggregate } \\
\left(\mathrm{kg} / \mathrm{m}^{3}\right)\end{array}$ & $\begin{array}{c}\text { Lightweight } \\
\text { aggregate } \\
\left(\mathrm{kg} / \mathrm{m}^{3}\right)\end{array}$ & $\begin{array}{c}\text { Fine } \\
\text { aggregate } \\
\left(\mathrm{kg} / \mathrm{m}^{3}\right)\end{array}$ & $\begin{array}{l}\text { Water } \\
\left(\mathrm{kg} / \mathrm{m}^{3}\right)\end{array}$ & $\begin{array}{l}\mathrm{w} / \mathrm{c} \\
\text { ratio }\end{array}$ \\
\hline $\mathrm{KC}$ & - & 806 & - & - & 645 & - & 645 & 282 & 0.35 \\
\hline KF1 & 12.2 & 810 & - & - & 648 & - & 648 & 312 & 0.39 \\
\hline KF2 & 24.3 & 806 & - & - & 645 & - & 645 & 338 & 0.42 \\
\hline CF2 & - & 297 & 74 & - & 1068 & - & 644 & 168 & 0.45 \\
\hline CF6 & - & 148 & 222 & - & 1068 & - & 584 & 168 & 0.45 \\
\hline CS6 & - & 148 & - & 222 & 1068 & - & 653 & 168 & 0.45 \\
\hline LF2 & - & 297 & 74 & - & - & 519 & 789 & 168 & 0.45 \\
\hline LF6 & - & 148 & 222 & - & - & 519 & 733 & 168 & 0.45 \\
\hline LS6 & - & 148 & - & 222 & - & 519 & 813 & 168 & 0.45 \\
\hline
\end{tabular}

TABLE 2: Mixture-independent elastic properties of concrete ingredients.

\begin{tabular}{lccc}
\hline Phase & $\begin{array}{c}\text { Bulk } \\
\text { modulus }(K) \\
{[\mathrm{GPa}]}\end{array}$ & $\begin{array}{c}\text { Shear } \\
\text { modulus }(\mu) \\
{[\mathrm{GPa}]}\end{array}$ & Source \\
\hline $\mathrm{CH}$ & 36.04 & 15.21 & {$[11]$} \\
Clinker & 107 & 49.38 & {$[11]$} \\
Fiber (kenaf) & 18.6 & 5.3 & {$[2]$} \\
Sand (quartz) & 35.92 & 25.8 & {$[32]$} \\
Lightweight aggregate & 7.14 & 6.5 & {$[43]$} \\
Coarse aggregate & 22.22 & 16.8 & {$[43]$} \\
\hline
\end{tabular}

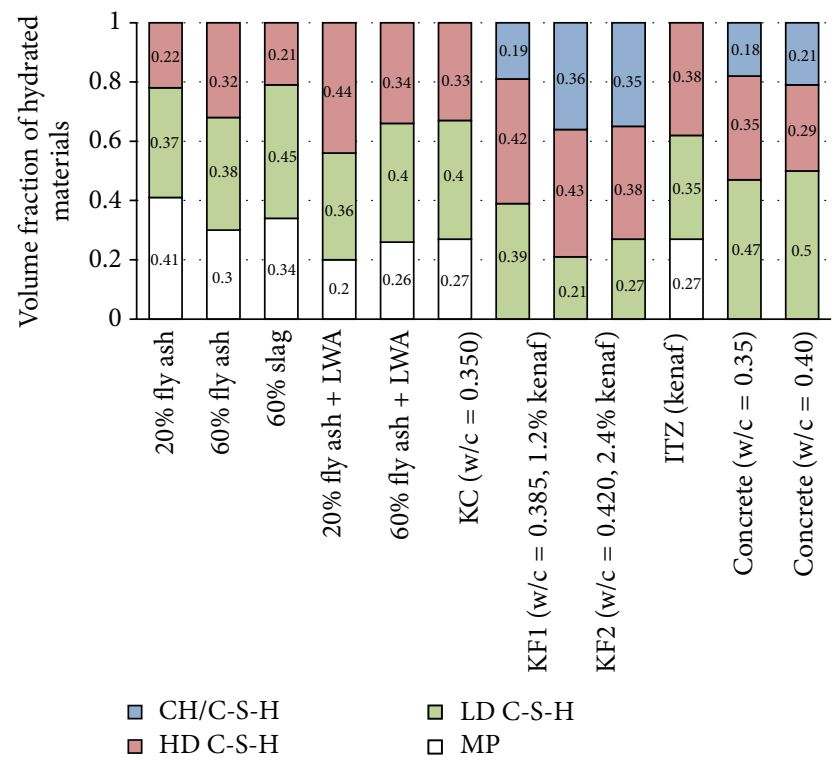

Figure 7: Hydration products of tested samples [7, 8, 38, 40].

\section{Results: Upscaling the Elastic Properties}

Figures 8 and 9 show the predicted elastic and shear modulus of the samples in different scale levels. The graphs

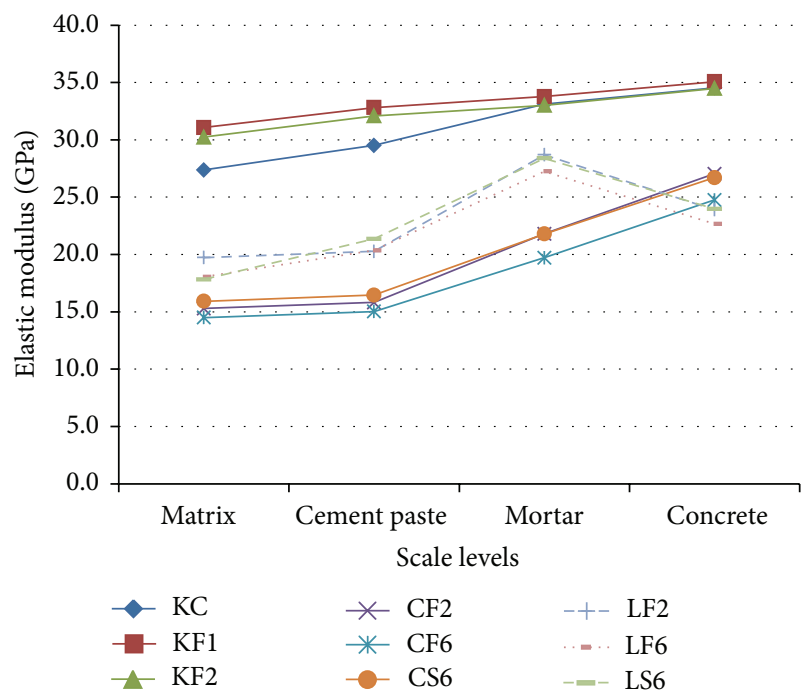

FIGURE 8: Predicted elastic modulus of the samples in different scale levels.

demonstrate how the properties change from one scale to another depending on volume fraction and mechanical properties of the ingredients. For the samples with conventional coarse aggregate, the properties increase with upscaling, whereas, for the samples with lightweight aggregate, properties increase up to level II then decrease because of lightweight aggregate's low elastic modulus contribution in scale level III.

The results of independent large-scale experiments were compared with model predictions for all the samples. Elastic modulus of CF2, CF6, CS6, LF2, LF6, and LS6 using conventional uniaxial unloading test was obtained by McCoy et al. [39] whereas ultrasonic test was used to determine the elastic modulus of KC, KF1, and KF2. There were no shear tests for kenaf samples, so shear moduli were calculated by knowing elastic modulus and assuming the Poisson's ratio $(v)$ of 0.3. According to Sorelli et al. [42] Poisson's ratio for hydrated products is around 0.24 . Using this assumption, 


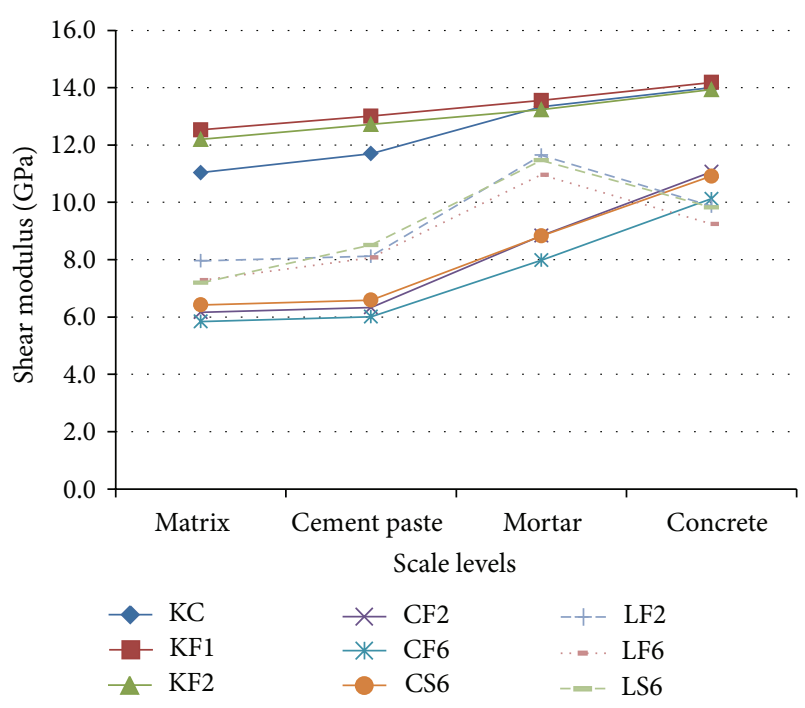

Figure 9: Predicted shear modulus of the samples in different scale levels.

the macroscopic Poisson's ratio for the samples was found by multiscale modeling, it was around 0.23 for all the samples.

The predicted elastic and shear modulus using NI technique and homogenization are plotted with respect to the measured ones from the uniaxial unloading test in Figures 10 and 11 . The dashed lines indicate $\pm 10 \%$ error. Predicted values almost coincide with uniaxial test measurements. Error for both CF6 and LF6 are higher than other samples; a possible reason can be high volume of fly ash in these samples which caused more error in the predictions. As the percentage of SCMs in mixture increases, the influence of other scale levels, for example, microcracks and porosities which were not characterized directly in this method, in multiscale modeling increases. Another reason could be because of inconsistency in large scale experimental results for these two samples; elastic modulus of LF6 was reported to be higher than CS6. More research is needed to refine predictions of the elastic mechanical properties of concrete incorporating high percentage of SCMs using micromechanics.

The overall agreement is an indication of the capability of the multistep homogenization method for sustainable concrete materials. Despite some errors between predicted and measured elastic modulus, the model is able to correctly predict trends and can serve as a tool to help in optimization of the concrete composition towards desired properties.

\section{Summary and Conclusions}

In this paper, multiscale modeling for three types of sustainable concretes was established using micromechanics and implemented for prediction of elastic properties. Different micromechanical homogenization schemes were addressed and applied to the modeling. Simplifications, including considering all the inclusions spherical, and limitations, such as not including microcracks inherent in concrete microstructure in the modeling of the implemented model, were

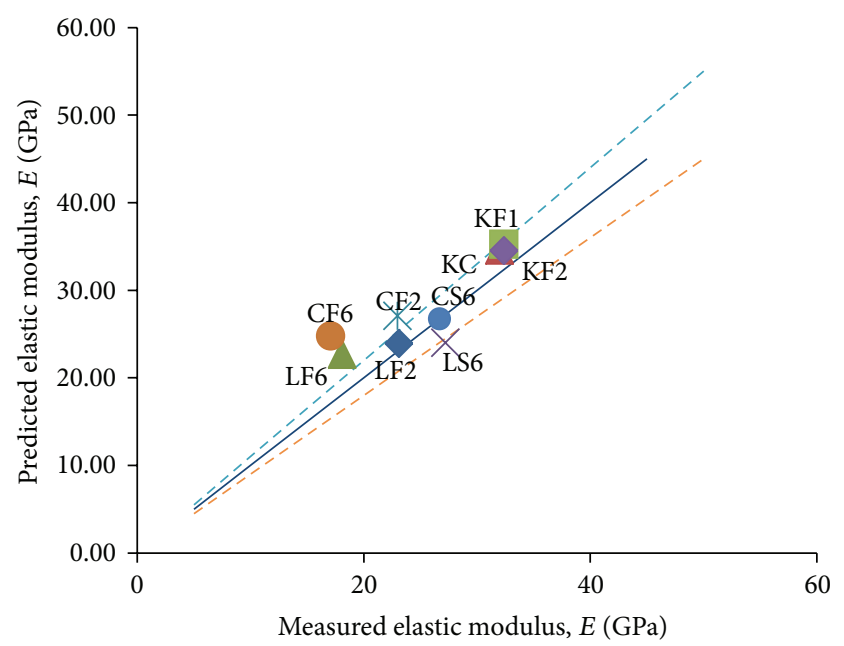

FIGURE 10: Predicted elastic modulus versus measured from experiment.

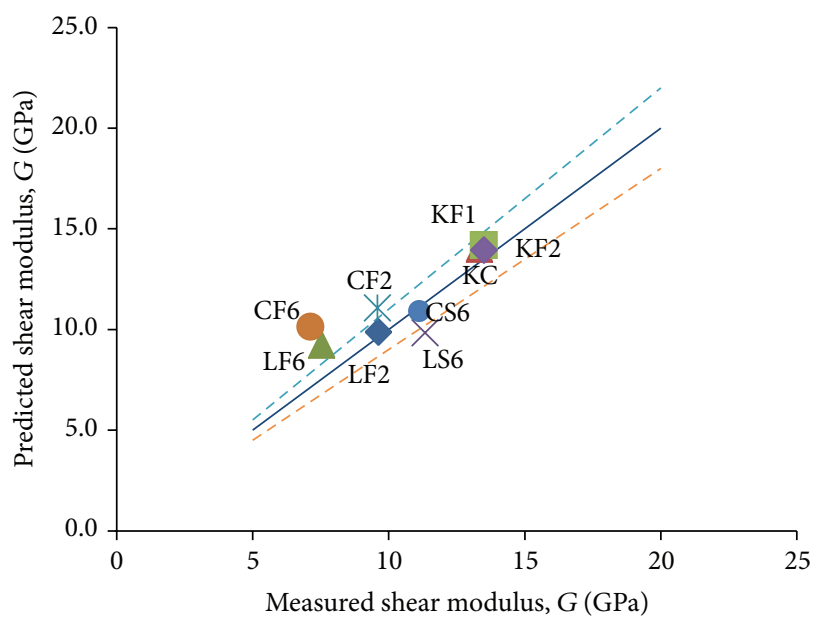

Figure 11: Predicted shear modulus versus measured from experiment.

presented. Incorporating inherent microcracks or ITZ effect as a weak boundary layer in cement microstructure could help to predict the macroscopic behavior more precisely and is suggested as future work. Despite any limitations, this multiscale investigation is a leap forward in application of stiffness homogenization models in sustainable cementbased materials.

In general, measured elastic properties obtained at the macroscopic scale and predicted from upscaled indentation modulus were consistent. This consistency forms an argument in favor of the proposed micromechanical modeling along with measured nanomechanical properties by nanoindentation technique to predict elastic properties of the sustainable concretes. This may serve for advanced elastic properties prediction, and more generally as a powerful and rational tool for material-to-structure optimization. 


\section{Conflict of Interests}

The authors declare that there is no conflict of interests regarding the publication of this paper.

\section{References}

[1] T. R. Naik, "Sustainability of concrete construction," ASCE Practice Periodical on Structural Design and Construction, vol. 13, no. 2, pp. 98-103, 2008.

[2] A. Elsaid, M. Dawood, R. Seracino, and C. Bobko, "Mechanical properties of kenaf fiber reinforced concrete," Construction and Building Materials, vol. 25, no. 4, pp. 1991-2001, 2010.

[3] American Concrete Institute, Use of Fly Ash in Concrete, ACI 232.2R, American Concrete Institute, Farmington Hills, Mich, USA, 2003.

[4] "Ground Granulated Blast Furnace Slag as a Cementitious Constituent in Concrete," ACI 233R, American Concrete Institute, Farmington Hills, Mich, USA, 2011.

[5] "ACI Manual, (308-213) R-13: Report on internally cured concrete using prewetted absorptive lightweight aggregates," 2013.

[6] S. Chandra and L. Berntsson, Lightweight Aggregate Concrete, Noyes, New York, NY, USA, 2002.

[7] V. Z. Zadeh and C. P. Bobko, "Nanomechanical investigation of internal curing effects on sustainable concretes with absorbent aggregates," in Proceedings of the 5th Biot Conference on Poromechanics (BIOT '13), pp. 1625-1634, July 2013.

[8] V. Z. Zadeh and C. P. Bobko, "Nanomechanical characteristics of lightweight aggregate concrete containing supplementary cementitious materials exposed to elevated temperature," Construction and Building Materials, vol. 51, pp. 198-206, 2014.

[9] G. Constantinides and F. J. Ulm, "The effect of two types of C$\mathrm{S}-\mathrm{H}$ on the elasticity of cement-based materials: results from nanoindentation and micromechanical modeling," Cement and Concrete Research, vol. 34, no. 1, pp. 67-80, 2004.

[10] S. Diamond, "Cement paste microstructure-an overview at several levels," in Hydraulic Cement Pastes: Their Structure and Properties, Proceedings of a Conference at Sheffield, pp. 2-31, University of Sheffield, Sheffield, UK, 1976.

[11] G. Constantinides and F.-J. Ulm, "The nanogranular nature of C-S-H," Journal of the Mechanics and Physics of Solids, vol. 55, no. 1, pp. 64-90, 2007.

[12] J. J. Chen, L. Sorelli, M. Vandamme, F.-J. Ulm, and G. Chanvillard, "A coupled nanoindentation/SEM-EDS study on low water/cement ratio portland cement paste: Evidence for C-S$\mathrm{H} / \mathrm{Ca}(\mathrm{OH}) 2$ nanocomposites," Journal of the American Ceramic Society, vol. 93, no. 5, pp. 1484-1493, 2010.

[13] H. M. Jennings, "A model for the microstructure of calcium silicate hydrate in cement paste," Cement and Concrete Research, vol. 30, no. 1, pp. 101-116, 2000.

[14] V. Zanjani Zadeh, Nanomechanics and multiscale modeling of sustainable concretes [Ph.D. dissertation], North Carolina State University, 2013.

[15] J. D. Eshelby, "The determination of the elastic field of an ellipsoidal inclusion, and related problems," Proceedings of the Royal Society. London. Series A. Mathematical, Physical and Engineering Sciences, vol. 241, pp. 376-396, 1957.

[16] J. D. Eshelby, "The elastic field outside an ellipsoidal inclusion," Proceedings of the Royal Society A: Mathematical, Physical and Engineering Sciences, vol. 252, pp. 561-569, 1959.
[17] J. D. Eshelby, "Elastic inclusions and inhomogeneities," in Progress in Solid Mechanics, N. I. Snedden and R. Hill, Eds., vol. 2, pp. 89-104, North Holland, 1961.

[18] Z. Hashin and S. Shtrikman, "On some variational principles in anisotropic and non-homogeneous elasticity," Journal of the Mechanics and Physics of Solids, vol. 10, pp. 335-342, 1962.

[19] Z. Hashin and S. Shtrikman, "A variational approach to the theory of the elastic behaviour of polycrystals," Journal of the Mechanics and Physics of Solids, vol. 10, pp. 343-352, 1962.

[20] W. Voigt, "Theoretische studien uber die elastizitatsverhaitnisse der krystalle," Abhandlungen der Königlichen Gesellschaft der Wissenschaften zu Göttingen, Mathematisch-Physikalische Klasse, vol. 34, no. 1, 47 pages, 1887.

[21] A. Reuss, "Berchnung der Fliessgrenze von Mischkristallen auf grund der plastizitatsbedingung fur einkristalle," Zeitschrift für Angewandte Mathematik und Mechanik, vol. 9, no. 49, 1929.

[22] G. I. Taylor, "Plastic strain in metals," Journal of the Institute of Metals, vol. 62, pp. 307-324, 1938.

[23] R. Hill, "Elastic properties of reinforced solids," Journal of the Mechanics and Physics of Solids, vol. 11, no. 5, pp. 357-372, 1963.

[24] A. V. Hershey, "The elasticity of an isotropic aggregate of anisotropic cubic crystals," Journal of Applied Mechanics, vol. 21, pp. 236-240, 1954.

[25] E. Kröner, "Computation of the elastic constants of polycrystals from constants of single crystals," Zeitschriftfür Physik, vol. 151, pp. 504-518, 1958 (German).

[26] T. Mori and K. Tanaka, "Average stress in matrix and average elastic energy of materials with misfitting inclusions," Acta Metallurgica, vol. 21, no. 5, pp. 571-574, 1973.

[27] A. Bensoussan, J.-L. Lions, and G. Papanicolaou, Asymptotic Analysis for Periodic Structures, North-Holland, 1978.

[28] E. Sanchez-Palencia, Non-Homogeneous Media and Vibration Theory, Springer, Berlin, Germany, 1980.

[29] P. Suquet, Elements of Homogenization for Inelastic Solid Mechanics, Springer, 1987.

[30] E. J. Garboczi and D. P. Bentz, "Computer-based models of the microstructure and properties of cement-based materials," in Proceedings of the 9th International Congress Chemistry of Cements, pp. 3-15, New Delhi, India, 1992.

[31] L. Dormieux, A. Molinari, and D. Kondo, "Micromechanical approach to the behavior of poroelastic materials," Journal of the Mechanics and Physics of Solids, vol. 50, no. 10, pp. 2203-2231, 2002.

[32] F.-J. Ulm, G. Constantinides, and F. H. Heukamp, "Is concrete a poromechanics materials? - a multiscale investigation of poroelastic properties," Materials and Structures, vol. 37, no. 1, pp. 4358, 2004.

[33] V. Šmilauer and Z. Bittnar, "Microstructure-based micromechanical prediction of elastic properties in hydrating cement paste," Cement and Concrete Research, vol. 36, no. 9, pp. 17081718, 2006.

[34] C. Pichler, R. Lackner, and H. A. Mang, "A multiscale micromechanics model for the autogenous-shrinkage deformation of early-age cement-based materials," Engineering Fracture Mechanics, vol. 74, no. 1-2, pp. 34-58, 2007.

[35] J. Qu and M. Cherkaoui, Fundamentals of Micromechanics of Solids, John Wiley \& Sons, Hoboken, NJ, USA, 2006.

[36] E. Herve and A. Zaoui, " $n$-Layered inclusion-based micromechanical modelling," International Journal of Engineering Science, vol. 31, no. 1, pp. 1-10, 1993. 
[37] A. Elsharief, M. D. Cohen, and J. Olek, "Influence of aggregate size, water cement ratio and age on the microstructure of the interfacial transition zone," Cement and Concrete Research, vol. 33, no. 11, pp. 1837-1849, 2003.

[38] V. Z. Zadeh and C. P. Bobko, "Nano-mechanical properties of internally cured kenaf fiber reinforced concrete using nanoindentation," Cement and Concrete Composites, vol. 52, pp. 9-17, 2014.

[39] B. C. McCoy, M. L. Leming, and R. Seracino, "Crack density and elastic properties of sustainable concretes," ACI Materials Journal, vol. 111, no. 1, pp. 13-21, 2014.

[40] V. Zanjani Zadeh and C. P. Bobko, "Nanoscale mechanical properties of concrete containing blast furnace slag and fly ash before and after thermal damage," Cement and Concrete Composites, vol. 37, no. 1, pp. 215-221, 2013.

[41] P. K. Mehta and P. J. M. Monteiro, Concrete: Microstructure, Properties, and Materials, Prentice-Hall, New York, NY, USA, 3rd edition, 2006.

[42] L. Sorelli, G. Constantinides, F.-J. Ulm, and F. Toutlemonde, "The nano-mechanical signature of ultra high performance concrete by statistical nanoindentation techniques," Cement and Concrete Research, vol. 38, no. 12, pp. 1447-1456, 2008.

[43] M. Alexander and S. Mindess, Aggregates in Concrete, Taylor \& Francis, New York, NY, USA, 2005. 

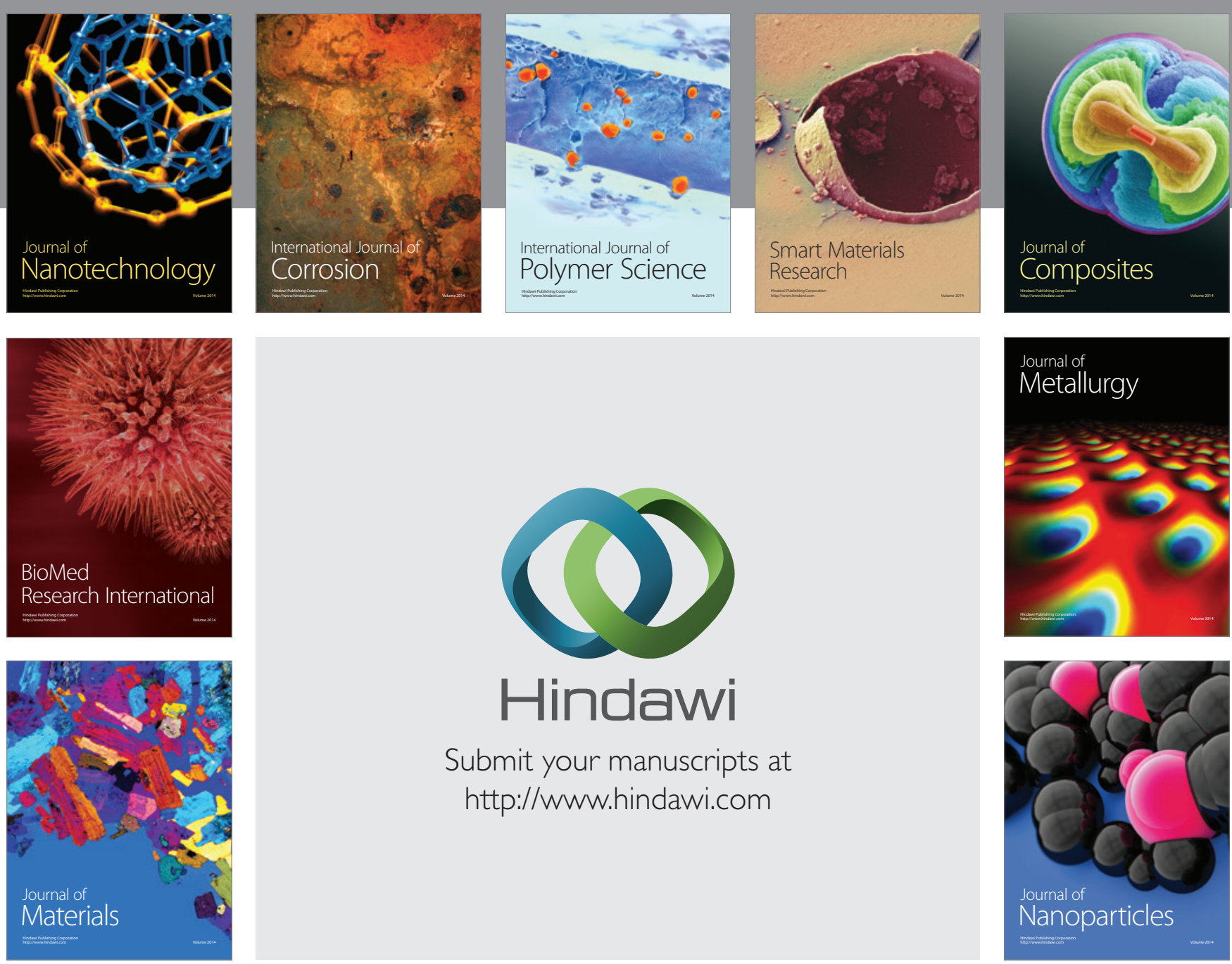

Submit your manuscripts at http://www.hindawi.com
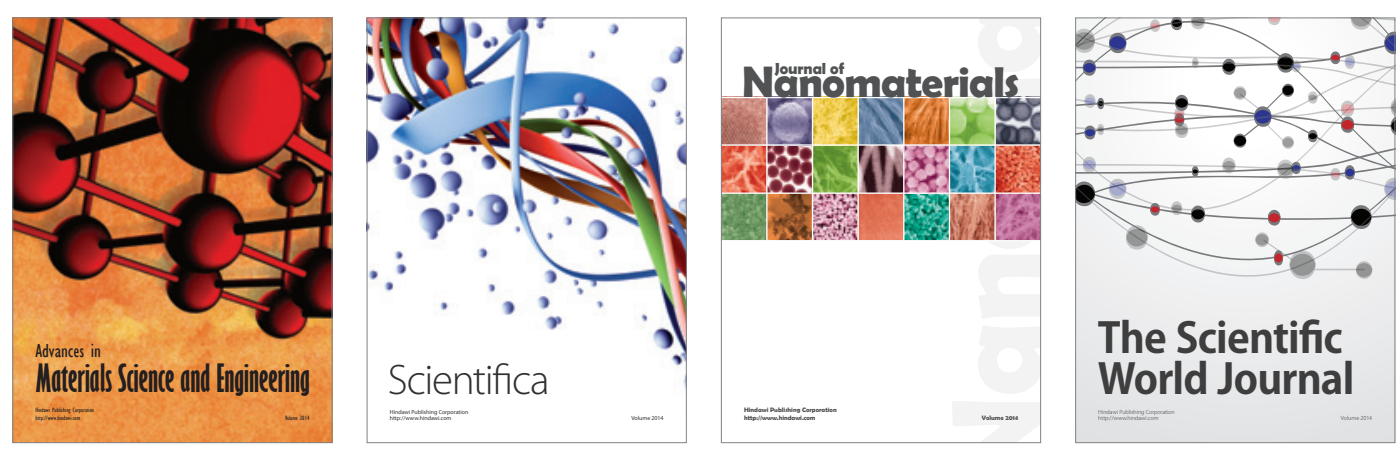

\section{The Scientific World Journal}
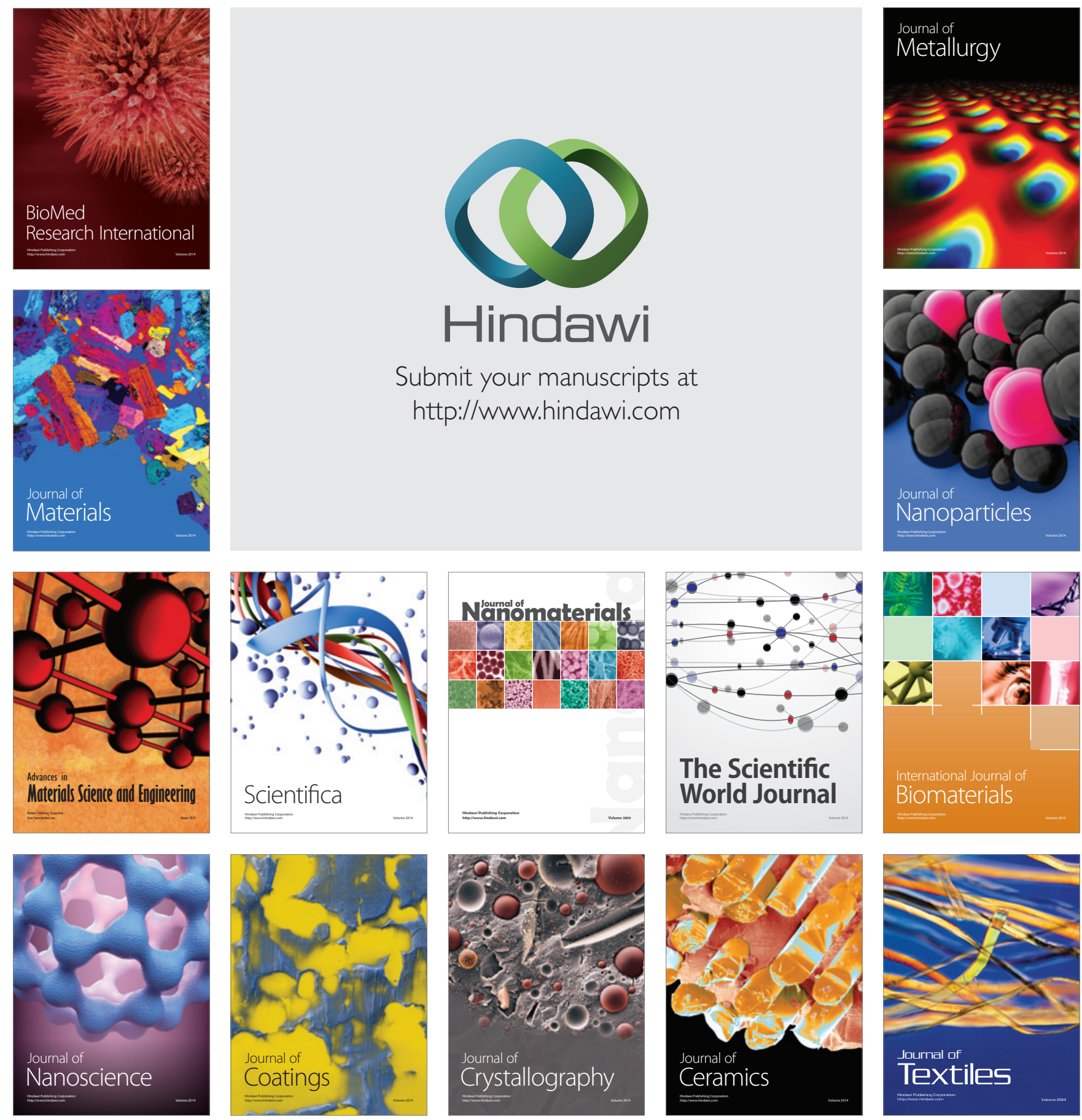